\title{
Screening for Food Insecurity in the Primary Care Setting: Type of Visit Matters
}

J Gen Intern Med 36(12):3907-9 DOI: $10.1007 / \mathrm{s} 11606-020-06474-\mathrm{X}$

(C) Society of General Internal Medicine 2021

\section{INTRODUCTION}

In 2018 over $11 \%$ of American households experienced food insecurity (FI), a condition of limited or uncertain access to food, putting those individuals at higher risk of chronic diseases including diabetes, obesity, depression, and hypertension. ${ }^{1}$

Primary care providers can help address patients' FI, but screening protocols vary widely, and standards of practice have not been identified. The likelihood that a patient will screen positively for FI may depend on the setting, timing, and format in which screening is performed. Many clinics screen for FI at annual health maintenance exams (HME), even though socioeconomically disadvantaged groups are less likely to attend routine health checks. ${ }^{2}$

We performed a retrospective cohort study to evaluate disparities in FI screening and prevalence based on visit type and patient demographics at a community health center serving low-income patients in Southeast Michigan.

\section{METHODS}

We obtained de-identified electronic health record data for all adult patient visits at the Ypsilanti Health Center between March 2018 and February 2019. This clinic employed a two-question FI screener. ${ }^{3}$ Patients aged 18-25 were excluded in order to eliminate visits with pediatricians from the analysis.

Characteristics of the screened and non-screened visits were compared using cross-tabulations and chi-squared tests. We assessed for differences in categorical variables using Pearson's chi-square and assessed the likelihood of being screened and having FI using logistic regression (R, Australia, 2013).

Received August 21, 2020

Accepted December 14, 2020

Published online January 19, 2021

\section{RESULTS}

Table 1 displays the characteristics of the sample population stratified by screening status. $24.5 \%$ of all visits were screened, and among all screened visits, $22.8 \%$ screened positive for FI (5.6\% of the total study population). After adjustment, (Table 2) screening was much more likely to occur at HME (OR 3.0, 95\%CI: 2.8-3.3) or New Patient (NP) visits (OR 1.8, 95\%CI: 1.4-2.2) than return visits. Hispanic patients were less likely to be screened than white patients (OR: 0.58 , 95\%CI 0.49-0.69). Elderly patients were more likely to be screened compared to adults aged 25-64 (OR 1.7, 95\%CI: $1.5-1.9)$.

Compared to white patients, non-Hispanic Black patients had 2.0 higher odds (95\% CI: 1.7-2.4) of screening positive for FI. Women had 1.9 higher odds (95\% CI: 1.6-2.3) of screening positive. FI was more prevalent among those with public insurance (OR 1.7, 95\% CI: 1.4-2.0) compared to private insurance. FI was less prevalent among HME visits (OR 0.82 , 95\%CI: $0.68-0.98$ ) and among urgent care visits (OR $0.48,95 \%$ CI: $0.26-0.89$ ) compared to return visits.

\section{DISCUSSION}

At this community health center, FI was less common among patients attending HME visits, though patients were three times more likely to be screened there than any other visit type. FI was more common among nonHispanic Black patients, females, and those with public insurance. These findings suggest that a screening process in which questionnaires are completed only at annual visits will not fully capture FI, particularly among at-risk populations.

These findings do not account for access to healthcare, screening method, or repeat visits, all of which could impact screening efficacy and are important to consider.

These results suggest that FI screening ought to align with AAFP recommendations ${ }^{4}$ for all patients regardless of visit type, and for patients who are at highest risk of being socioeconomically disadvantaged, whether due to race, insurance type, or sex. These findings are especially relevant in light of evidence of very high rates of FI, and income-based disparities in FI brought about by the COVID-19 pandemic. ${ }^{5}$ Screening only during new visits and health maintenance examinations may exacerbate 
Table 1 Sample Characteristics Representing All Adult Visits to YHC from March 2018 to February 2019

\begin{tabular}{|c|c|c|c|c|}
\hline & All visits & Not screened & Screened & $p$ value \\
\hline & $N=15,103$ & $N=\mathbf{1 1 , 4 0 1}$ & $N=3702$ & \\
\hline & $N(\%)$ & $N(\%)$ & $N(\%)$ & \\
\hline Visit type & & & & $<0.0001$ \\
\hline Health maintenance exam & 2661 (17.6) & $1510(13.2)$ & $1151(31.1)$ & \\
\hline New patient visit & $412(2.7)$ & $288(2.5)$ & $124(3.3)$ & \\
\hline Return visit & $11,659(77.2)$ & $9313(81.7)$ & $2346(63.4)$ & \\
\hline Urgent & $367(2.4)$ & $287(2.5)$ & $80(2.2)$ & \\
\hline Age & & & & $<0.0001$ \\
\hline 25-65 years & $12,992(86.0)$ & $9999(87.7)$ & $2993(80.8)$ & \\
\hline $66-100$ years & $2111(14.0)$ & $1402(12.3)$ & 709 (19.2) & \\
\hline Sex & & & & 0.06 \\
\hline Male & $5097(33.7)$ & $3800(33.3)$ & $1297(35.0)$ & \\
\hline Race & & & & $<0.0001$ \\
\hline Non-Hispanic Black & $6235(41.3)$ & $4752(41.7)$ & $1483(40.1)$ & \\
\hline Non-Hispanic White & $6187(41.0)$ & $4533(39.8)$ & $1654(44.7)$ & \\
\hline Hispanic & $1173(7.8)$ & $982(8.6)$ & $191(5.2)$ & \\
\hline Other or mixed race & $1049(6.9)$ & $787(6.9)$ & $262(7.1)$ & \\
\hline Insurance type & & & & 0.03 \\
\hline Private & $8087(53.5)$ & $6044(53.0)$ & $2043(55.2)$ & \\
\hline Public & $5883(39.0)$ & 4499 (39.5) & $1384(37.4)$ & \\
\hline Uninsured & $78(0.5)$ & $66(0.6)$ & $12(0.3)$ & \\
\hline
\end{tabular}

Table 2 Predictors of Food Insecurity Screening and Food Insecurity Prevalence Among Patients at YHC

\begin{tabular}{|c|c|c|c|}
\hline Predictor & Odds ratio & $95 \% \mathrm{CI}$ & $p$ value \\
\hline \multicolumn{4}{|c|}{ Odds of being screened for food insecurity during health center visit $(N=14,619)$} \\
\hline \multirow{2}{*}{\multicolumn{4}{|c|}{$\begin{array}{l}\text { Visit type } \\
\text { Return visit }\end{array}$}} \\
\hline & & & \\
\hline Health maintenance exam & 3.0 & $2.8-3.3$ & $<0.0001$ \\
\hline New patient visit & 1.8 & $1.4-2.2$ & $<0.0001$ \\
\hline Urgent visit & 1.1 & $0.86-1.4$ & 0.41 \\
\hline \multicolumn{4}{|l|}{ Age } \\
\hline Age $25-65$ & {$[\operatorname{Ref}]$} & & \\
\hline Age $>65$ & 1.7 & $1.5-1.9$ & $<0.0001$ \\
\hline \multicolumn{4}{|l|}{ Sex } \\
\hline Male & {$[\operatorname{Ref}]$} & & \\
\hline Female & 1.1 & $0.97-1.1$ & 0.19 \\
\hline \multicolumn{4}{|l|}{ Race } \\
\hline Non-Hispanic White & [Ref] & & \\
\hline Non-Hispanic Black & 0.94 & $0.86-1.0$ & 0.14 \\
\hline Hispanic & 0.58 & $0.49-0.69$ & $<0.0001$ \\
\hline Other or mixed race & 0.95 & $0.82-1.1$ & 0.56 \\
\hline \multicolumn{4}{|l|}{ Insurance type } \\
\hline Private & [Ref] & & \\
\hline Public & 0.90 & $0.83-0.98$ & 0.02 \\
\hline None & 0.59 & $0.31-1.1$ & 0.10 \\
\hline \multicolumn{4}{|c|}{ Odds of food insecurity among screened patients $(N=3590)$} \\
\hline \multirow{2}{*}{\multicolumn{4}{|c|}{ Visit type }} \\
\hline Return visit & & & \\
\hline Health maintenance exam & 0.82 & $0.68-0.98$ & 0.03 \\
\hline New patient visit & 1.4 & $0.91-2.1$ & 0.13 \\
\hline Urgent Visit & 0.48 & $0.26-0.89$ & 0.02 \\
\hline \multicolumn{4}{|l|}{ Age } \\
\hline Agge 25-65 & [Ref] & & \\
\hline Age $>65$ & 0.40 & $0.31-0.52$ & $<0.0001$ \\
\hline \multicolumn{4}{|l|}{ Sex } \\
\hline Male & [Ref] & & \\
\hline Female & 1.9 & $1.6-2.3$ & $<0.0001$ \\
\hline \multicolumn{4}{|l|}{ Race } \\
\hline Non-Hispanic White & [Ref] & & \\
\hline Non-Hispanic Black & 2.0 & $1.7-2.4$ & $<0.0001$ \\
\hline Hispanic & 0.78 & $0.52-1.2$ & 0.23 \\
\hline Other or mixed race & 0.79 & $0.55-1.1$ & 0.19 \\
\hline \multicolumn{4}{|l|}{ Insurance type } \\
\hline Private & [Ref] & & \\
\hline Public & 1.7 & $1.4-2.0$ & $<0.0001$ \\
\hline None & 3.1 & $0.96-10.2$ & 0.06 \\
\hline
\end{tabular}


existing disparities in care based on race, income, or gender by reducing the likelihood of detection.

Acknowledgments: The authors would like to thank the faculty and staff at the Ypsilanti Health Center for their collaboration and their eagerness to facilitate this study.

Megan R. McLeod, MD MS ${ }^{1,2}$

Anita Vasudevan, $M D^{1,3}$

Stephen Warnick Jr, $M D^{4}$

Julia A. Wolfson, PhD, MPP 5,6

${ }^{1}$ University of Michigan Medical School,

Ann Arbor, MI, USA

${ }^{2}$ Internal Medicine Residency Program, Ronald

Reagan UCLA Medical Center, UCLA Health,

Los Angeles, CA, USA

${ }^{3}$ Internal Medicine Residency Program, Yale,

New Haven, CT, USA

${ }^{4}$ Beaumont Family Medicine Residency,

Grosse Pointe, MI, USA

${ }^{5}$ Department of Health Management and Policy, University of Michigan School of Public Health,

Ann Arbor, MI, USA

${ }^{6}$ Department of Nutritional Sciences, University of Michigan School of Public Health,

Ann Arbor, MI, USA

Corresponding Author: Megan R. McLeod, MD MS; Internal Medicine Residency Program, Ronald Reagan UCLA Medical Center, UCLA Health, Los Angeles, CA, USA (e-mail: mrmcleod@mednet.ucla.edu).
Authors Contribution Study concept and design: McLeod, Vasudevan

Data acquisition: Vasudevan, McLeod

Analysis or interpretation of data: McLeod

Drafting of the manuscript: McLeod

Figures: McLeod

Critical revision of the manuscript: all authors

Final approval: all authors

Funding MM was supported by TL1TROO2242.

\section{Compliance with Ethical Standards:}

Conflict of Interest: The authors declare that they do not have a conflict of interest.

\section{REFERENCES}

1. Coleman-Jensen A, et al. Household food security in the United States in 2018 (ERR-270). US Department of Agriculture, Economic Research Service. 2019;47:1-47.

2. Wolf ER, et al. Gaps in well-child care attendance among primary care clinics serving low-income families. Pediatrics. 2018;142(5):e20174019.

3. Hager ER, et al. Development and validity of a 2-item screen to identify families at risk for food insecurity. Pediatrics. 2010;126:e26-e32.

4. Patil SP, Craven K, Kolasa K. Food insecurity: how you can help your patients. Am Fam Physician. 2018;98:143-145.

5. Wolfson JA, Leung CW. Food insecurity and COVID-19: disparities in early effects for US adults. Nutrients. 2020;12:1648.

Publishers Note Springer Nature remains neutral with regard to jurisdictional claims in published maps and institutional affiliations. 